\title{
MS29-02 | Influence Of A Hydrogen Bond on Optical Properties of Materials
}

Krawczuk, Anna (Jagiellonian University, Faculty of Chemistry, Cracow, POL)

The correlation between the crystal structure and physical properties of a given material has long been a subject of many studies. One of the key features of designing efficient multifunctional materials is to use specific building blocks and/or synthons in order to increase a desired effect in a certain crystallographic direction. For example, to obtain efficient optical devices it is necessary to use highly polarizable functional groups which will form strong hydrogen bonds and promote high optical effect, e.g high refractive index. It is thus crucial to get a precise information on the influence of hydrogen bonds on the group polarizabilities which in turn should give an answer which hydrogen bonds will mostly contribute to the physical effect. This in turn could be very helpful for the crystal engineering purpose when designing new optically effective materials. 